\title{
THE AMPLITUDES OF WAVES TO BE EXPECTED IN SEISMIC PROSPECTING
}

\section{B. GUTENBERG}

\begin{abstract}
A method of calculating the amplitudes of bodily waves in earthquakes is applied to a study of the amplitudes of longitudinal waves produced by an artificial explosion. Formulae are given for calculating the energy of the reflected longitudinal wave arriving at the surface. The percentage of energy reflected at a discontinuity increases rapidly after the angle of incidence exceeds the critical refraction angle. However, no corresponding large amplitudes are recorded by the instruments. Instead, the maximum amplitudes of reflected waves are found near the shot point. This is because the ground movement is due both to the arriving wave and to the wave reflected downward from the ground. The amplitude of this movement depends, besides, on the rate of change of the angle of incidence with distance. It is pointed out that the same methods can be applied to a dipping bed, and that the amplitudes of the reflected waves are in general slightly larger in the up-dip than in the down-dip direction.
\end{abstract}

A method for calculating the amplitudes of bodily waves in earthquakes was suggested by K. Zoeppritz in Igo8. The formulae to be used have been worked out by the author in the years following the death of Zoeppritz, and the first application to earthquake waves was made by L. Geiger and B. Gutenberg. ${ }^{2}$ The method has since been used successfully in several investigations. The following is an application of this method in the study of amplitudes of longitudinal waves produced by an artificial explosion.

We suppose that the energy is produced at a point $A$ (Fig. I) on the surface of the earth, that the same amount of energy is radiated in all directions, and that there is no change in properties in horizontal directions. No assumptions are made concerning the vertical direction; the velocity may increase or decrease either gradually or suddenly with depth. We consider two rays which leave the point $A$ (Fig. I) under the angles of incidence $i_{1}$ and $i_{2}$ which differ by the small amount $d i$. After refractions and reflections the rays arrive finally at the distances $\Delta_{1}$ and $\Delta_{2}$ at the surface which differ by the small amount $d \Delta$.

According to our assumptions, through each unit area of a sphere around $A$ having the very small radius $r$, the same amount $e$ of energy

1 Balch Graduate School of the Geological Sciences, California Institute of Technology, Pasadena, California; Contribution No. 202.

${ }^{2}$ Karl Zoeppritz, Ludwig Geiger und Beno Gutenberg, Ueber Erdbebenwellen V.

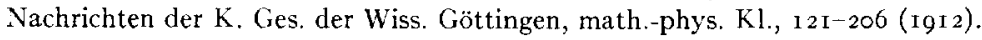


is passing. The rays which leave $A$ between the cones given by the angles $i_{1}$ and $i_{2}$ intersect the sphere in the zone

$$
I=2 \pi r h=2 \pi r^{2} d i \sin i .
$$

The total energy flowing between the two cones just mentioned is $e I$. At each reflection and refraction of the rays, the energy is split into the longitudinal and transverse reflected and refracted waves. If $F$

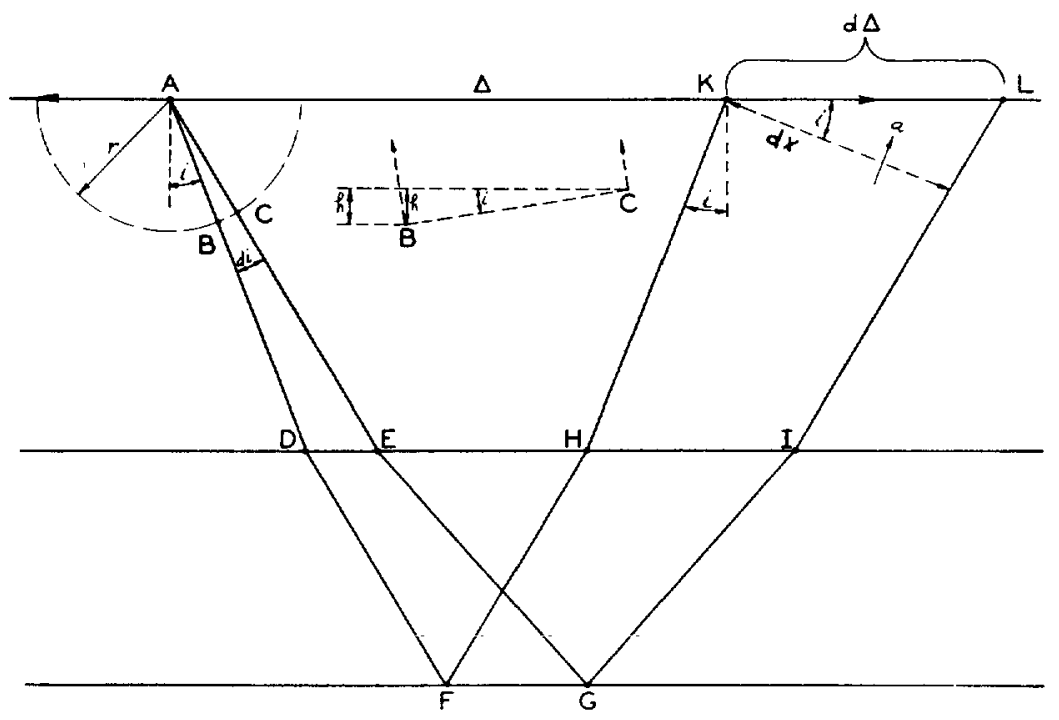

FIG. I

is the product of all factors giving the ratio of the energy flux along the path under consideration after and before the refraction (at $D E$ and $H I$ in Fig. $\mathbf{r}$ ) or reflection (at $F G$ ), the total energy arriving finally at the surface of the earth is $F e I$. This energy is spread over a zone at the distance $\Delta$ from $A$ of the width $d x$ measured perpendicular to the energy flux and with the area $2 \pi \Delta d x=2 \pi \Delta d \Delta \cos i$. The energy per unit of the area is therefore

$$
E=\frac{F e I}{2 \pi \Delta d \Delta \cos i}=r^{2} e \frac{F d i \sin i}{\Delta d \Delta \cos i}=C \frac{F}{\Delta} \frac{d i}{d \Delta} \tan i
$$

where $C$ is a constant for each explosion depending on the energy generated. The amplitude $a$ of the ground motion in the wave approaching the surface is for a given period proportional to $\sqrt{E}$. At 
the surface, a longitudinal and a transverse wave are reflected, and the displacement of a particle of the surface is due not only to the amplitude of the arriving wave, but also to the movements in the two reflected waves. The direction of the ground movement, therefore, in general differs from the direction of the arriving waves, and the displacement of the ground does not only depend on the amplitude of the arriving wave but also on the angle of incidence and Poisson's ratio. If $B$ is the factor with which $E$ is to be multiplied in order to find the amplitude $A$ of the ground in a given component, we find finally the fundamental equation:

$$
A=c B \sqrt{F\left|\frac{d i}{d \Delta}\right| \frac{\tan i}{\Delta}}
$$

where $c$ depends on the original energy and the period of the wave and where $i$ is the angle of incidence at the distance $\Delta$, where the wave is recorded.

For a given value of Poisson's ratio, $B$ can be calculated as a function of the angle $i .^{3}$ Supposing Poisson's ratio $\sigma=0.27^{2}$, which is a good approximation in most cases, we find the following values of $B$ for the horizontal and vertical components of the ground movements, if the angle of incidence $i$ is given:

$\begin{array}{ccccccccccccc}i & 0 & 5 & 10 & 20 & 30 & 40 & 50 & 60 & 70 & 80 & 85 & 90^{\circ} \\ \mathrm{B}_{\text {hor }} & 0.00 & 0.19 & 0.39 & 0.75 & \mathrm{I} .08 & \mathrm{I} .36 & \mathrm{I} .57 & \mathrm{1} .66 & \mathrm{I} .6 \mathrm{I} & \mathrm{I} .26 & 0.83 & 0.00 \\ \mathrm{~B}_{\text {vert }} & 2.00 & \mathrm{I} .99 & \mathrm{I} .97 & \mathrm{I} .86 & \mathrm{I} .7 \mathrm{I} & \mathrm{1} .50 & \mathrm{I} .27 & \mathrm{I} .04 & 0.8 \mathrm{I} & 0.54 & 0.34 & 0.00\end{array}$

If the horizontal component is recorded, the first line must be used, in the case of a vertical component the second line.

To calculate the factor $F$, we must know the energy ratio between the reflected or refracted longitudinal wave and the arriving wave at each point of reflection or refraction. Formulae to calculate these ratios of energies have been given by $\mathrm{C}$. G. Knott, ${ }^{4}$ while $\mathrm{K}$. Zoepprit $z^{5}$ has developed formulae to calculate the amplitudes. The calculations based on these equations show that for vertical incidence the ratio of the reflected energy $E_{r}$ to the arriving energy $E_{a}$ is given by

$$
\left[\frac{E_{r}}{E_{a}}\right]_{i=0}=\frac{\left(\rho_{2} V_{2}-\rho_{1} V_{1}\right)^{2}}{\left(\rho_{2} V_{2}+\rho_{1} V_{1}\right)^{2}}
$$

${ }^{3}$ For details see for example: B. Gutenberg, Handbuch der Geophysik, vol. 4, pp. $4^{2-} 48$.

${ }^{4}$ C. G. Knott, Reflexion and refraction of seismic waves, Phil. Mag. 48, 64 (I 899).

${ }^{5}$ K. Zoeppritz, Ueber Erdbebenwellen VII b, Nachr. K. Ges. der Wiss. Göttingen, math.-phys. K1. (I919), p. 57 . 
where $\rho_{1}$ and $\rho_{2}$ are the densities in the two media, while $V_{1}$ and $V_{2}$ are the corresponding velocities of longitudinal waves. In exchanging the indices in the formula one finds that the ratio is the same whether the wave is reflected at the upper or lower side of the boundary.

In general, $E_{r} / E_{a}$ decreases slightly with increasing $i$, reaches a minimum and increases then slowly. In this whole interval under normal circumstances, the larger part of the energy, frequently over $90 \%$, is to be found in the refracted longitudinal wave. When $\sin i$ at the reflecting layer approaches the value given by the ratio of the longitudinal velocity in the first layer over the longitudinal velocity in the second layer, beyond which no refracted longitudinal wave exists, the energy of the reflected wave begins to increase more rapidly. In the early experiments with the reflection method the region where these rays reach the surface were looked for, as large amplitudes of the reflected waves were expected there, but usually no such effect was found.

This is in perfect agreement with the theory, as has been shown by calculations in which conditions have been supposed corresponding about to the velocities in the San Joaquin Valley northwest of Bakersfield. Under the assumptions made in that paper ${ }^{6}$ the increase in the amplitudes of the reflected waves due to the larger percentage of reflected energy occurs at distances of several thousand meters from the shot point, and the amplitudes there usually are noticeably smaller than the amplitudes of the reflected waves near the shot point. The reason for this result is to be found in the other quantities of equation (3). As may be seen from the tabulation for $B$, its value decreases with increasing $i$ or with increasing distance. In the case of waves reflected from the bottom of a homogeneous layer it can be easily found from a sketch that

$$
\tan i=\frac{\Delta}{2 d}, \text { and therefore } \frac{d i}{d \Delta}=\frac{\cos ^{2} i}{2 d}=\frac{2 d}{4 d^{2}+\Delta^{2}} .
$$

With these values, we find in our special case

$$
A_{c}=c B \frac{\cos i}{2 d} \sqrt{\frac{E_{r}}{E_{a}}}=\frac{c B}{\sqrt{4 d^{2}+\Delta^{2}}} \sqrt{\frac{E_{r}}{E_{a}}} .
$$

At the large distances where the maximum of $E_{r} / E_{a}$ occurs, $\cos i$ is

${ }^{6} \mathrm{~B}$. Gutenberg, On some problems concerning the seismic field method. Beiträge zur angewandten Geophysik, in press. 
very small and prevents the occurrence of large amplitudes there together with the smaller values of $B$.

In the neighborhood of the shot point, $B$ has the value 2 for records of vertical amplitudes, $\cos i$ is close to I, $E_{r} / E_{a}$ is given by (4) where usually we can neglect the small differences in density. We find, therefore, that in the neighborhood of the shot point the amplitudes of the reflected waves are approximately proportional to $(\mathrm{I} / d)\left({ }_{2} V-V_{1}\right)$ $/\left(V_{2}+V_{1}\right)$, where $d$ is the depth of the reflecting layer and $V$ the velocities of longitudinal waves below $\left(V_{2}\right)$ and above $\left(V_{1}\right)$, it. A positive value of this expression $\left(V_{2}>V_{1}\right)$ corresponds to a movement of the ground in the same direction as in the original wave, downward in the case of an explosion, a negative value $\left(V_{2}<V_{1}\right)$ to a movement in the opposite direction.

If the refracting and reflecting layers have a dip, it is still possible to find the approximate amplitudes of the waves. In this case, the wave paths can be constructed in a similar way as indicated in Fig. $\mathrm{I}$, and the values of $d x$ and $i$ can be found graphically. In some cases investigated in this way it was found that the amplitudes of reflected waves are in general slightly larger in the direction opposite to the dip than in the direction of the dip. 